\title{
Lip Forensics for Criminal Identification
}

\section{P. Chandra Sekharan*}

Honorary Professor of Forensic Sciences, Dr. Ambedkar Law University, Chennai, India

\begin{abstract}
A careful glimpse of the lips instantly tells us the mood of the individual. The lips are the most expressive and the most mobile, or flexible facial feature, but in its normal relaxed state, it can be described quite accurately. Since the tendency is to watch the lips of another when he or she speaks, sight recognition of the lips should have a more lasting impression and the witness will be able to describe the lips better than any other facial feature. Thus the description of lips becomes an important aspect in facial recognition and identification.

The microstructure (grooves and wrinkles) contrary to the earlier findings, are not age-sable like finger prints, neither are they 'environmental-stable'. Therefore there may be some working disadvantages when lip prints of criminals are compared after a long lapse of time with the crime prints made earlier.

Nevertheless, the macrostructure, namely, the size of the lips, the shape of the oral fissure, upper and lower vermillion borders and protrusion of lips are age stable and environmental stable. Therefore the classification system of macrostructure can be potentially used for the processes of personal appearance identification. If witnesses can describe correctly the above five parameters, then it will easy to searching for the suspects could be narrowed down to one in 8640 .

The author has developed a universally applicable language to obviate the difficulty in using proper terminology by the witnesses and the inability of the investigating police to understand what the witnesses speak. The terminology developed by the author could easily be used by the witnesses and could easily be understood by the investigating police.
\end{abstract}

Keywords: Lip forensics; Cheiloscopy; Personal identification; Personal appearance identification; Sight; Recognition

\section{Introduction}

A careful glimpse of the lips instantly tells us the mood of the individual. The lips are the most expressive and the most mobile, or flexible facial feature, but in its normal relaxed state, it can be described quite accurately. Since the tendency is to watch the lips of another when he or she speaks, sight recognition of the lips should have a more lasting impression and the witness will be able to describe the lips better than any other facial feature. Thus the description of lips becomes an important aspect in facial recognition via lips.

This Paper is the result of the comprehensive research on lips carried out by the author in association with Dr TR Kumari initially during 1989-1993 and continued again until the recent few years, perhaps even until early 2012 to study at least one aspect namely to confirm our findings disproving the claim of the earlier researchers that the lip patterns (grooves and wrinkles) are permanent throughout the life of an individual [1-5].

In our work we studied lips as a whole and we termed the 'grooves and wrinkles' as microstructure of lips and the 'size and shape of the lips' as macrostructure. The objective of our work was therefore two folds taking into consideration, for the first time, the micro and macrostructures of the lips together and to evolve a comprehensive system so that the microstructure of the lips could be potentially used for personal identification and the macrostructure for personal appearance identification.

\section{Materials and Methods}

The basic materials for this study include 2063 Indian subjects selected at random from general population and 106 new born babies. Portrait photographs of 25 individuals taken at different stages of their lives extending up to sixty years were obtained to study the labial structures recorded in those photographs.

\section{Anatomy of lips}

Mouth would mean, in common usage, lips. Anatomically the mouth is defined as the oral cavity consisting of an outer, smaller part, the vestibule and an inner larger part, the oral cavity proper. Actually the lips externally bound the vestibule of the mouth and appear as a slit like space.

The lips can therefore be better described as two fleshy folds which surround the oral orifice and are formed externally of skin and internally of mucous membrane and these two layers enclose the muscle orbicularis oris, the labial vessels and nerves, areolar tissue, and numerous small labial salivary glands.

\section{Vermillion zone}

The lips are the junction between (i) the skin of the face which is parakeratinized stratified squamous epithelium and (ii) the mucosa of the oral cavity which is moist non-keratinized to parakeratinized stratified squamous epithelium. Between these two areas lies a transitional zone of reddish tissue known as the vermillion zone of

*Corresponding author: Dr. P. Chandra Sekharan, Honorary Professor of Forensic Sciences, Dr. Ambedkar Law University, Chennai, India, E-mail: pcsekharan2000@yahoo.com

Received November 20, 2012; Accepted December 29, 2012; Published December 31, 2012

Citation: Sekharan PC (2013) Lip Forensics for Criminal Identification. J Forensic Res S11: 007. doi:10.4172/2157-7145.S11-007

Copyright: ( 2013 Sekharan PC. This is an open-access article distributed unde the terms of the Creative Commons Attribution License, which permits unrestricted use, distribution, and reproduction in any medium, provided the original author and source are credited. 
the lip. It is this vermillion zone which is commonly denoted as 'lips' in forensic identification procedures. Vermillion zone is marked by various grooves and wrinkle as shown in Figure 1.

The biological phenomenon of the furrows present on the vermillion zone of human lips was first noticed and described as early as 1902 by Fischer, an anthropologist [6].

It was Yasuo Tsuchihashi [7] who first gave the anatomical name "Sulci Labiorum Roborum" to the wrinkles and grooves found on human lips. He gave the name "Figura Linearum Labiorum Robrorum" for the prints made by these Sulci (Figure 2). But Tsuchihashi has made no distinction between grooves and wrinkles. In our work we considered groves and wrinkles as separate entities.

\section{Pioneers in lip print identification}

Le Moyne Snyder [8] first suggested that wrinkles and grooves of the lips have the same individual characteristics as finger ridges. Santos [9] attempted classification of lip prints by dividing the grooves into two types and further subdividing the grooves into eight types. Suzuki and Tsuchihashi [10] classified the grooves into six types. Hassan et al. [11] suggested classification system of lip prints consisting of seven types.

Tsuchihashi and Suzuki examined 1364 persons at the Department of Forensic Odontology at Tokyo University and laid down certain basic principles for the study of lip prints. They established that the arrangements of lines on the vermillion zone of human lips are individualistic and unique for each human being [12]. This hypothesis has led to the conclusion that there is the possibility of using the arrangement of furrows on lips for the identification of a person. Tsuchihashi [12] also examined the hereditary factors in relation to lip prints. But none of these researchers studied in detail the size and shape of the lips in detail.

\section{Lip print evidence in criminal case}

It was Hungary in 1962 lip prints was used to identify a criminal. Lip traces were found on a glass door at the scene of a murder. It was established later that the murderer pushed the door open with his head because his hands were stained with blood. He therefore left a legible trace of his lips on the glass panel on the door. The lip print was compared with the lip prints of a suspect and found to be in agreement and thus the criminal was identified [13]. This made a beeline of researchers to rush to lip prints. These researchers compared lip prints to fingerprints and attempted blindly to attribute all tenets of fingerprint science to lip prints.

Finger ridges vis a vis Lip grooves.

We found that comparing the grooves on the lips to the epidermal ridges on the terminal segments of digits is an erroneous conception. The morphology of both is different. The epidermal ridges (also called 'Rugae') are formed on the surface of the digits as raised narrow ridges separated by fine but distinct parallel grooves, disposed in curved arrays. Profile of finger ridges vis-a-vis labial grooves are shown diagrammatically in Figure 3.

There is sufficient proof of the unchangeable nature of the ridge pattern on the digits. Since epidermal ridges are developed in the foetus during the third and fourth foetal months, the configurations and their component ridges enlarge keeping pace with the growth of hand and foot, but all their essential characters remain unchanged.
We cannot assume the same state of affairs in case of grooves and wrinkles in the vermillion zone of the lips even though the earlier researchers claim that they are permanent. In as much as the vermillion zone of the lips is subjected to contraction and expansion depending upon the several functions of the mouth, even at any one point of time, the pattern of grooves and wrinkles undergo temporary structural changes.

It has been proved by us that these changes tell upon the permanency of labial patterns. One significant finding in this study is that the microstructure (grooves and wrinkles) are not age-stable like finger prints. Neither they are 'environmental-stable'.

Therefore there may be some working disadvantages when lip prints of criminals are compared after a long lapse of time after the crime prints are made. The lip photographs of the same individual taken in 1952, 1970, 1985, 1993 and 2012 are shown in Figure 4.

Please note the appearance of cones in 1985 and 1993, where as in the 2012 photograph, there are no grooves but mostly wrinkles, belying the permanency theory proposed by all the earlier researchers.

Nevertheless, we have also studied grooves and wrinkles of all the 2169 subjects in detail. The earlier researchers described 'grooves and wrinkles' as one entity. In the present work grooves and wrinkles are identified as separate entities and distinct definitions given. The labial grooves are defined as

"Linear or curvy linear furrows or shallow depression found on the vermillion zone of the lips".

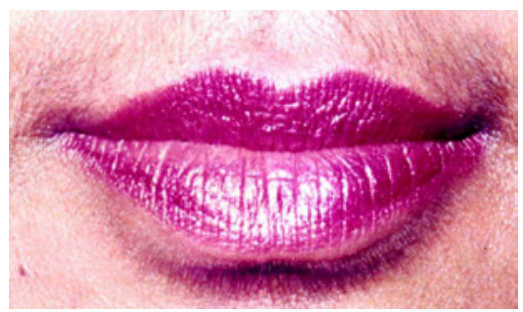

Figure 1: The vermillion zone marked by grooves and wrinkles.

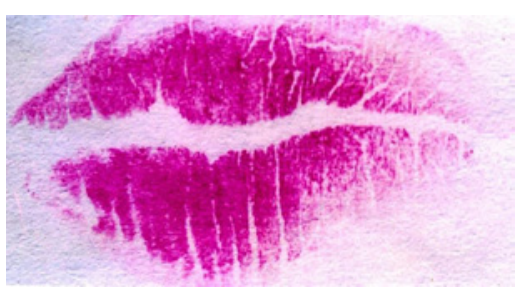

Figure 2: Lip prints made by sulci of the lips.

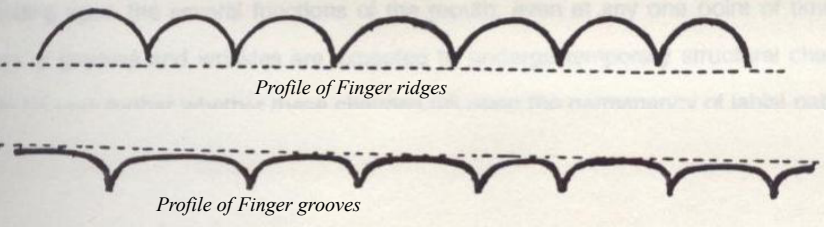

Figure 3: Diagrammatic profile of finger ridges versus labial grooves (Dotted line denotes the surface of the skin). 


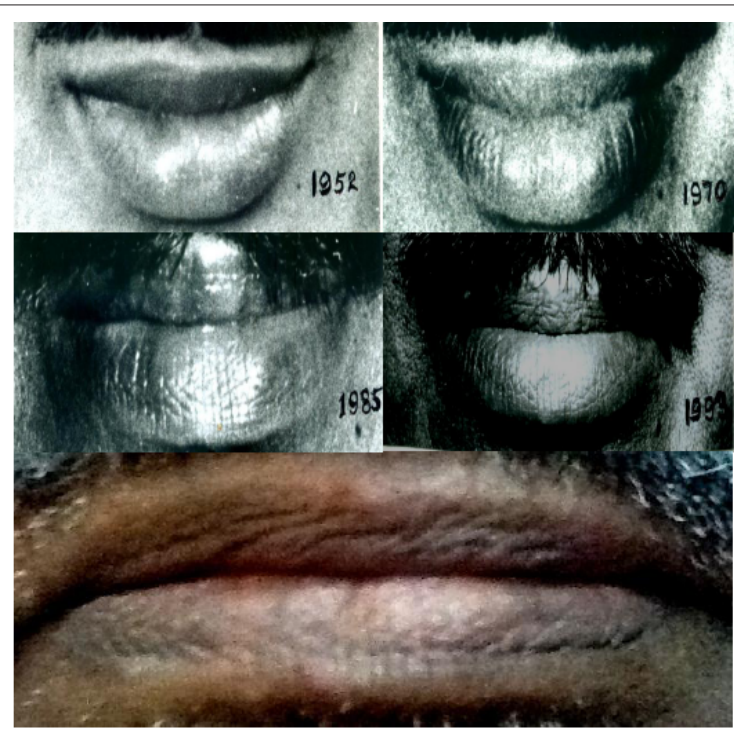

Figure 4: Lip Photographs of the same individual taken during 1952, 1970, 1985, 1993, 2012. Please note the gross change of microstructure in 2012

The labial wrinkles are defined as

"Shriveling appearance of the formation of corrugation in skin due to ageing"

Based on this definition, we have classified grooves into thirty two distinct types and wrinkles into eight distinct types and assigned to each type an internationally acceptable terminology [14]. But we concluded that microstructure of lips (lip prints) can be used only to a limited extent as trace evidence to identify criminals in the sense that test lip prints are obtained not after the lapse of three years or so after crime prints are noticed.

\section{Classification System for Labial Structures}

All classification systems rely on a limited number of descriptions and are based on class characteristics. In the classification system proposed for labial structures each defined class of lip contains configurations that can vary considerably in general appearance, while each class is specially defined with a nomenclature for the first time for the system used. There may be points in any classification system where it will be impossible to determine if a particular configuration is of one class or another. Also the differences between two classes cannot be expected to be so clear cut as that between parrots and crows. This is so in the system proposed for the structure for the lips.

The classification of lip pattern is therefore very simply the process of putting them into grouping of which all have some recognizable over all similarity. The classification of macrostructure of lips following the above process will be useful for investigating agencies in the personal appearance identification of suspects. The classification of the patterns of the microstructures (grooves and wrinkles) in lips in a similar fashion will be futile in as much as they are not permanent and the test prints of lips have to be made not after a long time after the crime print is noticed.

Thus in this study, classification systems are evolved both for microstructure and macrostructure; macrostructure for personal appearance identification and microstructure for personal identification. Personal appearance identification is an important area in identifying the suspects as per the descriptions of the witnesses. On the other hand, personal identification is connected with the identification of crime scene trace evidence, such as finger prints, foot prints, skeletal remains etc. which will lead to the identification of the individuals involved in crimes.

\section{Macrostructure of lips for identifying criminals by sight recognition}

The classification system evolved for personal appearance identification takes into account the macrostructure of lips as viewed in the (1) face norma frontalis and (2) face norma lateralis, because, the witnesses will describe the structure of lips in the frontal and lateral views of suspects' faces.

\section{Classification of macrostructure in lips norma frontalis}

The classification system for macrostructure is based on the size and shape of lips when the lips are viewed in the face norma frontalis; The shape of lips is further subdivided into three types. Thus four labial parameters are identified. They are:
(1) Size of lips
(2) Shape of Lips
a) Oral fissure
b) Upper vermillion border
c) Lower vermillion border

The size of the lips is determined by taking into consideration the maximum vertical dimension between the vermillion border and the oral fissure in both the upper and lower lips (Figure 5).

The thickness is measured between the upper most point in the upper vermillion border and the oral fissure in the case of upper lip and the same is measured between the bottom most point in the lower vermillion border and the oral fissure in the case of the lower lip.

The scrutiny of the lips of the 2063 subjects according to the vertical dimension of the lips resulted in identifying four types.

1. Thick

2. Thin

3. Thick Upper

4. Thin Upper

Based on the class characters, the above four types are defined as follows:

Thick (Type 1): In this type both the upper and the lower lips will be thick and be of the same size (Figure 6).

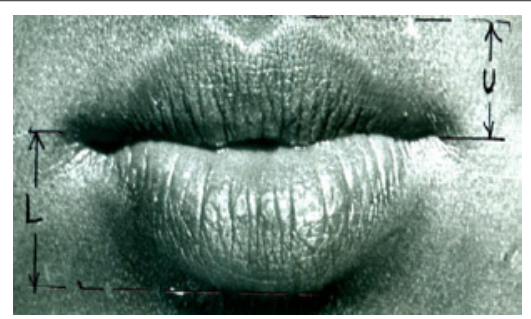

Figure 5: Thickness of lips: U-thickness of upper lip; L-Thickness of lower lip. 
Thin (Type 2): In this type both the upper and the lower lips will be thin and be of the same size (Figure 7).

Thick upper (Type 3): This type is the combinations with the upper lip thick and lower lip thin (Figure 8).

Thin upper: This type is the combination with the upper lip thin and the lower lip thick (Figure 9).

\section{Shape of lips}

The shape of lips is decided by the three factors viz. oral fissure; upper vermillion border; and lower vermillion border as shown in Figure 10.

\section{Oral fissure}

The oral fissure is the line of contact between the closed lips in its normal position. The photographs of the lips of all the 2063 subjects are scrutinized for differentiating the shapes of the oral fissure. Ten different types of configurations are identified to vary within limits and are termed as:
1. Straight
2. Notch
3. Upturned
4. Downturned
5. Sinuous
6. Drooping
7. Lenticular
8. Intersticed
9. Dimple
10. Cleft

All the above ten type of oral fissure are defined thus:

Straight (Type 1): In this category the line of contact will be a clearly defined straight line being perfectly horizontal in the normal position as shown below (Figure 11).

Notch (Type 2): The line of contact in this category will be clearly defined but on both sides of the line, notches will be found due to the presence of deep fissure on the closed lips (Figure 12).

Upturned (Type 3): The line of contact will be concave in shape in this type, with both ends upturned (Figure 13).

Downturned (Type 4): In this type the line of contact will be convex in shape with both the ends of the oral fissure downturned (Figure 14).

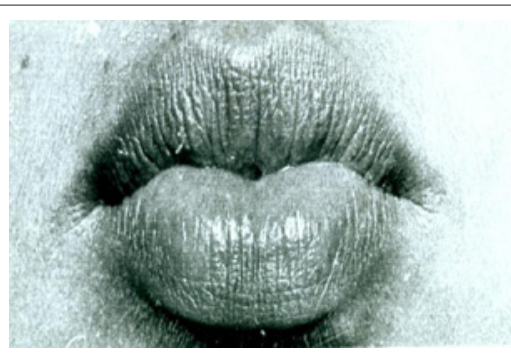

Figure 6: Thick lips (Type 1).
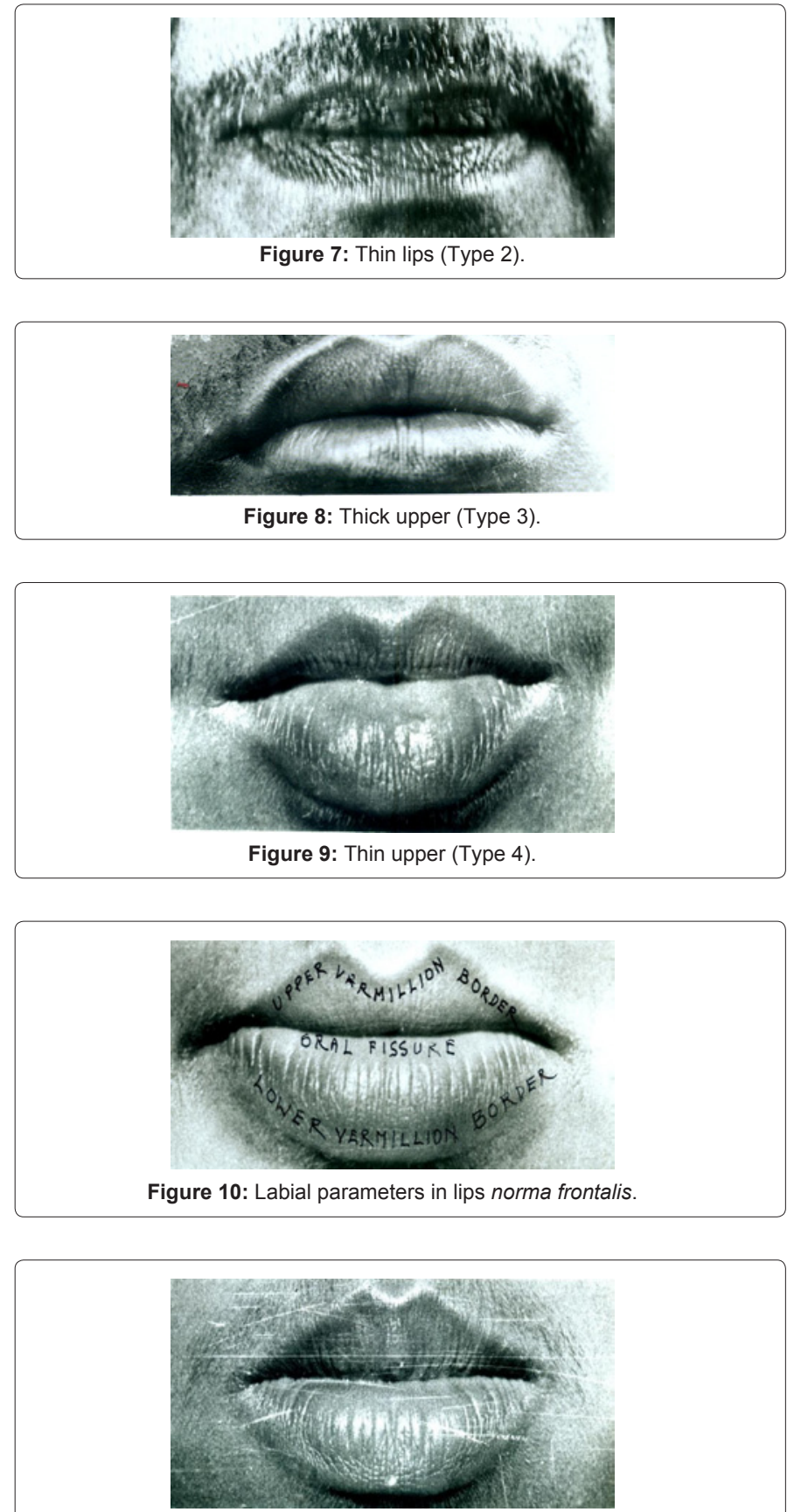

Figure 11: Oral Fissure: Straight (Type 1).

One should be careful in differentiating the downturned and upturned oral fissure, because, the shape of the oral fissure in the same individual will be viewed differently in two different postures of the face, when the head is flexed or extended. Figure below shows the shape of the oral fissure in the same individual in two different postures of the face (Figure 15).

Sinuous (Type 5): The line of contact will be curving gracefully as the winding of the road or the movement of the snake (Figure 16).

Drooping (Type 6): The line of contact will be circular and extending downwards beyond the commissure of the mouth (Figure 17). 
Lenticular (Type 7): In this category there won't be a single line of contact at all, since the lips will not be fully closed. The oral fissure will be in the form of biconvex slit while the teeth are shown (Figure 18).

Intersticed (Type 8): The line of contact will not be continuous due to incomplete closure of lips in certain lengths exhibiting one or more interstices (Figure 19).

Dimple (Type 9): In this category the oral fissure will end with a dimple at both the ends (Figure 20).

Cleft (Type 10): This is a congenital phenomenon in which the upper lip will be split and oral fissure will also be cut into two or three segments depending upon whether the cleft is unilateral or bilateral and will bend upwards to meet the upper vermillion border (Figure 21). It is estimated that the incidence of cleft lip in Indian population is 1 in 800 .

The oral fissure (Type 10) and as well as the upper vermillion border (Type 12) are termed as cleft. The two most common types of cleft lips are unilateral and bilateral clefts.

\section{Shape of upper vermillion border}

The upper vermillion border is defined as the border line separating the skin of the face below the nose and the vermillion zone of the upper lip. The shape of the upper vermillion border was found to vary within

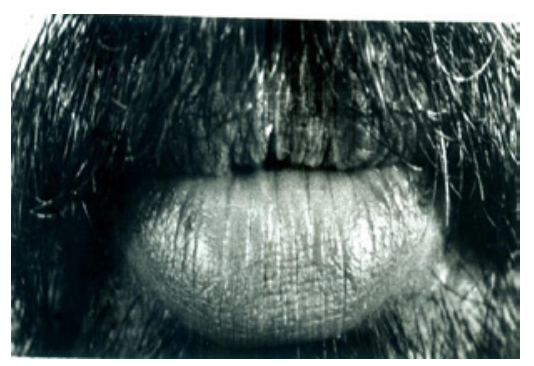

Figure 12: Oral Fissure: Notch (Type 2).

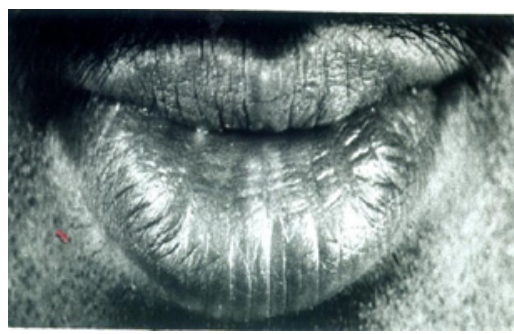

Figure 13: Oral Fissure: Upturned (Type 3).

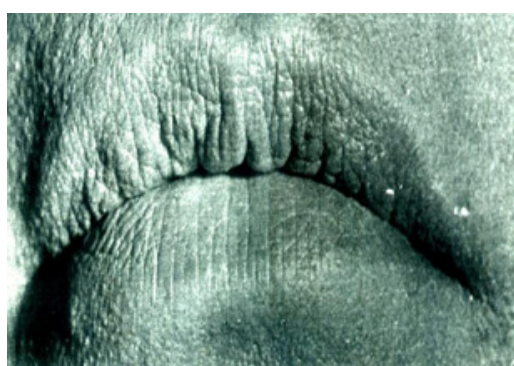

Figure 14: Fissure: Downturned (Type 4).

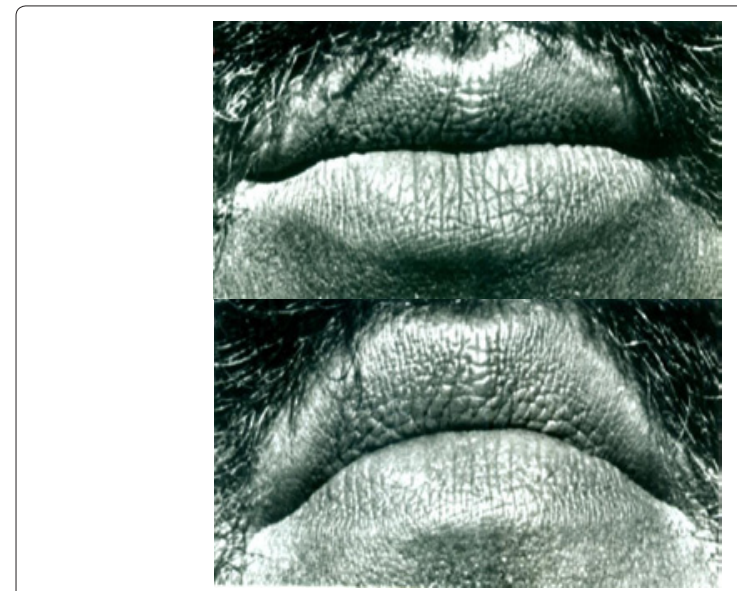

Figure 15: Oral fissure of the same individual in two different postures of the face.

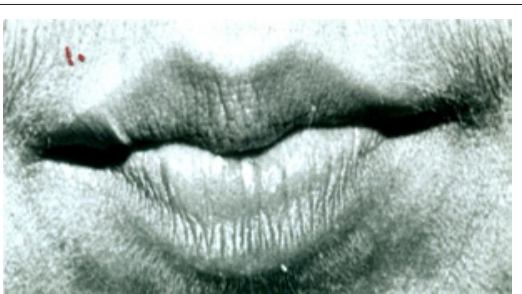

Figure 16: Oral Fissure: Sinuous (Type 5).

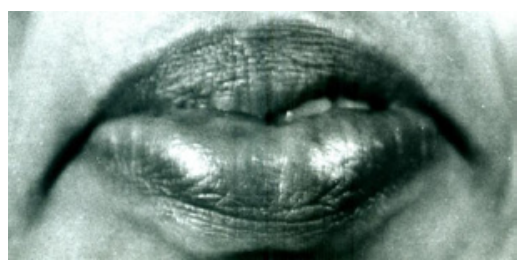

Figure 17: Oral Fissure: Drooping (Type 6).

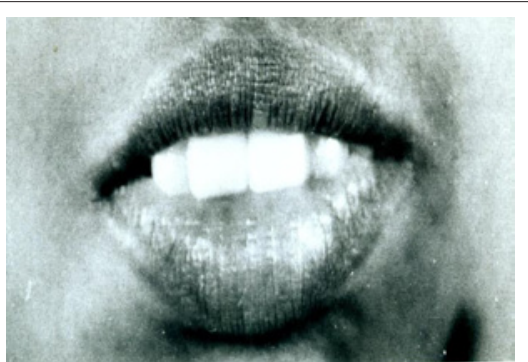

Figure 18: Oral Fissure: Lenticular (Type 7).

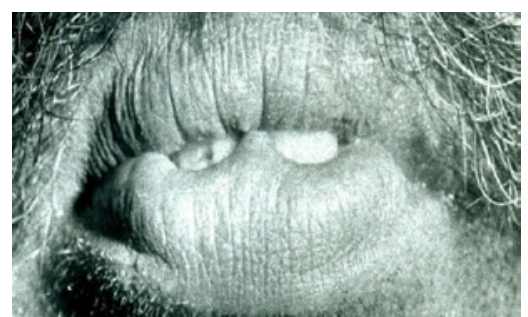

Figure 19: Oral Fissure: Intersticed (Type 8). 
limits and twelve types have been identified and they are termed as:

1. Twin peaks

2. Uneven peaks

3. Cantilever bridge

4. Suspension bridge

5. Butte

6. Double convex

7. Butterfly

8. Dome

9. Flying bird

10. Sine curve

11. Flat

12. Cleft

All the twelve types of upper vermillion border are defined as follows:

Twin peaks (Type 1): In this type the upper vermillion border will appear in the form of two adjacent peaks of even height with a ' $\mathrm{V}$ ' groove in the middle (Figure 22).

Uneven peaks (Type 2): The border line in this type will be in the form of two adjacent peaks but with uneven height. One will be taller than the other (Figure 23).

Cantilever bridge (Type 3): The border line in this case will be in the form of two peaks separated by a plateau (Figure 24).

Suspension bridge (Type 4): The border line will look like the upper border of the suspended bridge having two peaks with a concave meniscus in the centre (Figure 25).

Butte (Type 5): The border line in this case will be a hill with a flat top and steep sides (Figure 26).
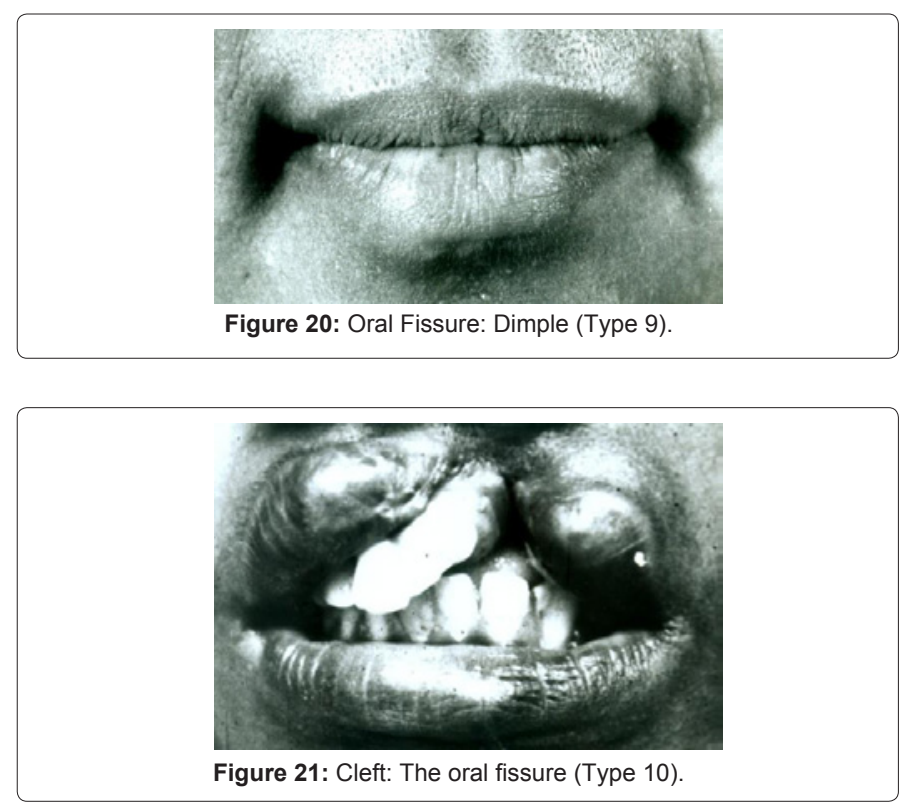
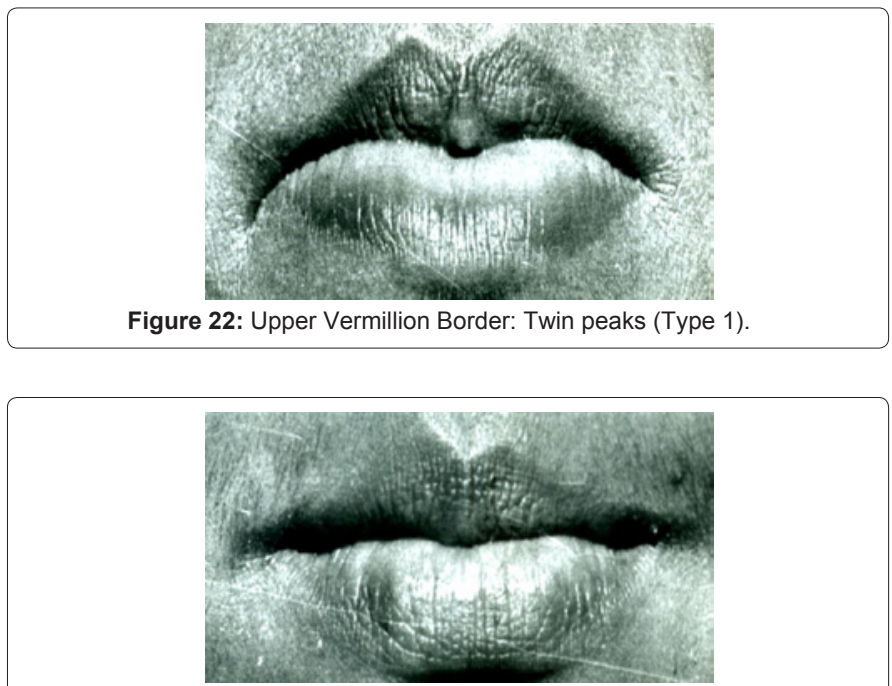

Figure 23: Upper Vermillion Border: Uneven peaks (Type 2).

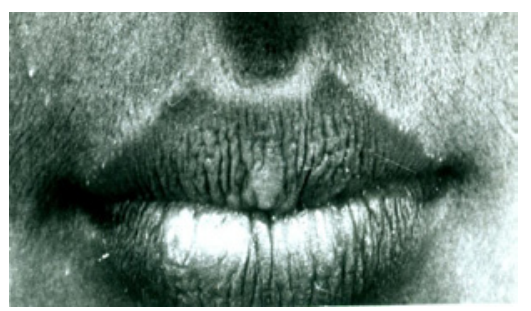

Figure 24: Upper Vermillion Border: Cantilever Bridge (Type 3).

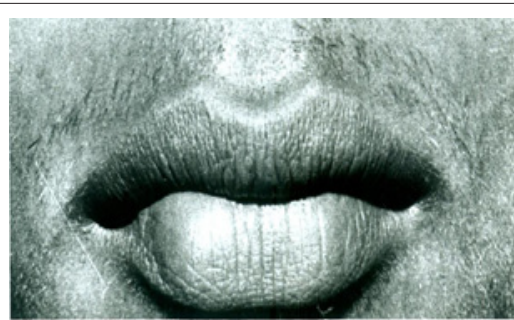

Figure 25: Upper Vermillion Border: Suspension bridge (Type 4).

Double convex (Type 6): The border line in this case will be in the shape of two convex curves joining at the median line (Figure 27).

Butterfly (Type 7): The border line in this case will resemble the spread wings of the butterfly with a pitch in the middle which rises on both sides and tapers down smoothly (Figure 28).

Dome (Type 8): The entire upper border line will appear in the form of an arc shaped dome (Figure 29).

Flying bird (Type 9): The upper border line will be in the shape of the wings of a flying bird (Figure 30).

Sine curve (Type 10): The upper border line will be in the form of a sine curve with two crests on sides and one trough in the middle (Figure 31).

Flat (Type 11): The upper border line will almost be flat, parallel to the oral fissure (Figure 32). 
Cleft (Type 12): The border line in this case will be cut into two or three segments depending upon whether the cleft is unilateral or bilateral and will bend downwards to meet the oral fissure (Figure 33).

\section{Shape of the lower vermillion border}

The lower vermillion border is the line separating the vermillion zone of the lower lip and the skin of the chin. The shapes of the lower vermillion border in all 2063 subjects were found to vary within limits into six types and they are termed as:

1. Apple bottom

2. Boat

3. Basin

4. Concave arc

5. Companulate

6. Flat

All the above six types are defined as follows:

Apple bottom (Type 1): The border line in this case will appear like the bottom of the apple with an elevated crest at the centre (Figure 34).

Boat (Type 2): In this case the border line will resemble the shape of a boat with a flat bottom (Figure 35).

Basin (Type 3): In this type, the border line will resemble the shape of a basin (Figure 36).

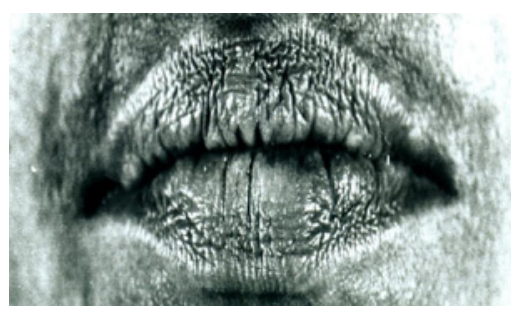

Figure 26: Upper Vermillion Border: Butte (Type 5).

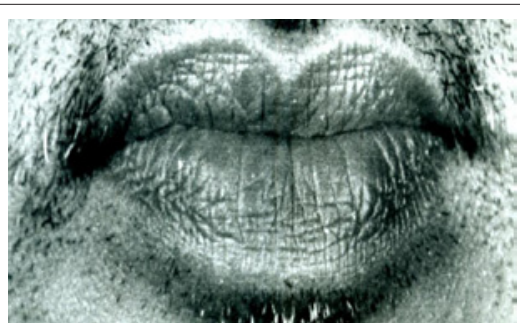

Figure 27: Upper Vermillion Border: Double convex (Type 6).

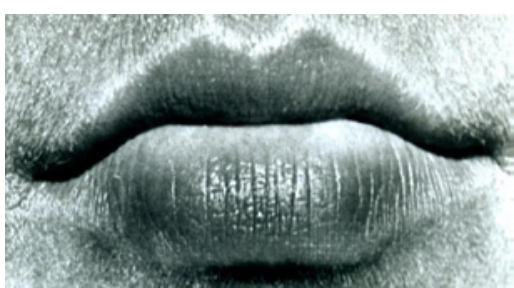

Figure 28: Upper Vermillion Border: Butterfly (Type 7)

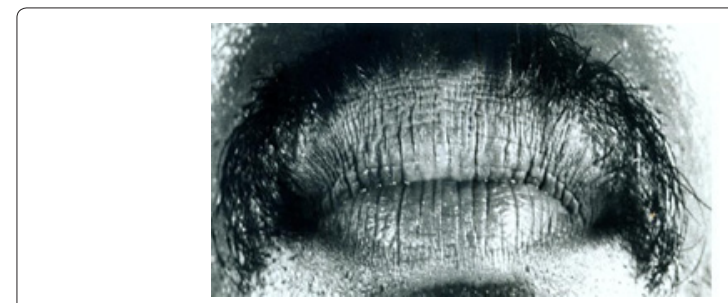

Figure 29: Upper Vermillion Border: Dome (Type 8).

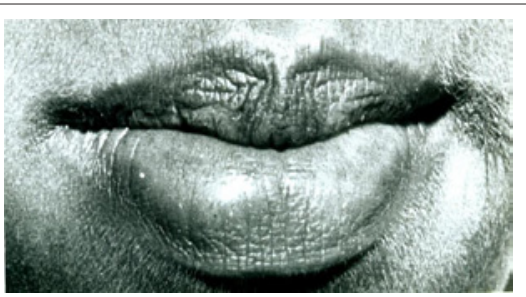

Figure 30: Upper Vermillion Border: Flying bird (Type 9)

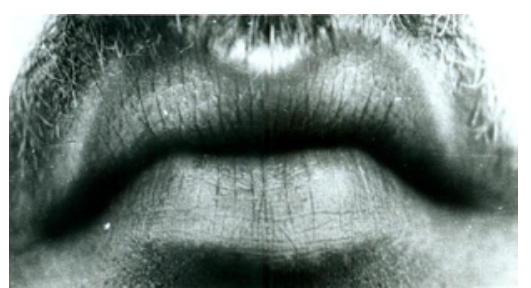

Figure 31: Upper Vermillion Border: Sine curve (Type 10)

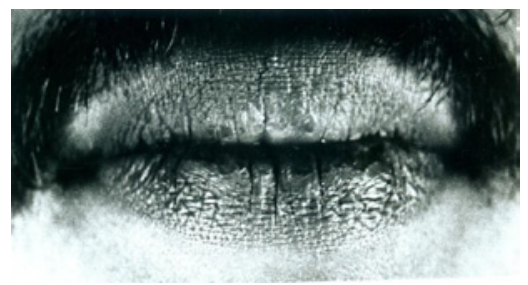

Figure 32: Upper Vermillion Border: Flat (Type 11).

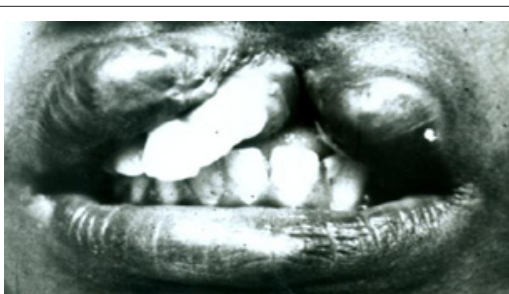

Figure 33: Cleft (Type 12).

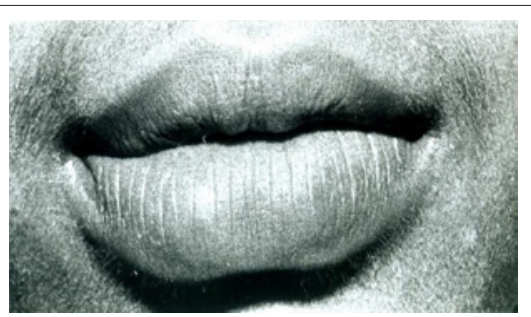

Figure 34: Lower Vermillion Border: Apple bottom (Type 1). 
Concave arc (Type 4): The border line in this case will be concave in shape (Figure 37).

Companulate (Type 5): In this case, the border line will be in the shape of a cup or an inverted bell (Figure 38).

Flat (Type 6): The border line in this type will almost be a straight line parallel to the oral fissure (Figure 39).

\section{Classification of macrostructure in lips norma lateralis (Protrusion)}

The classification system for macrostructure of lips norma lateralis is based on the protrusion of the lips with respect to each other. Three categories have been identified in this view given distinct definitions.

1. Even lips

2. Protruding upper lip

3. Protruding lower lip

Even lips (Type 1): In this category, the lips are evenly placed so that the line joining the extreme points in the vermillion zone of both the lips is vertical (Figure 40).

Protruding upper lip (Type 2): In this category, the upper lip will be protruding compared to the lower lip (Figure 41).

Protruding lower lip (Type 3): In this category the lower lip will be protruding compared to the upper lip (Figure 42)

Thus the macrostructure of lips norma lateralis can be divided only into three groups.

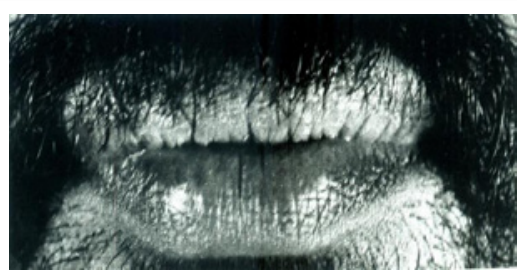

Figure 35: Lower Vermillion Border: Boat (Type 2).

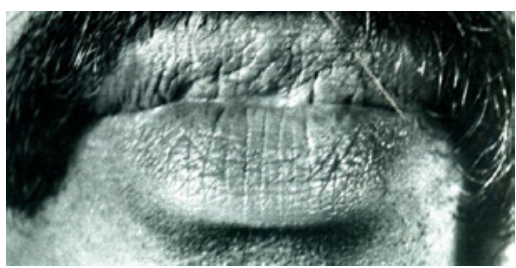

Figure 36: Lower Vermillion Border: Basin (Type 3).

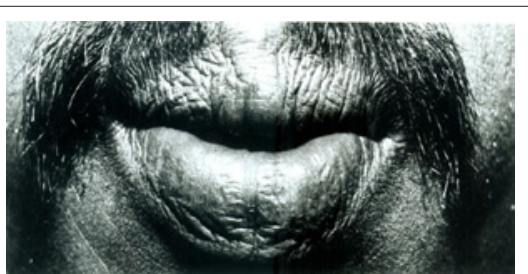

Figure 37: Lower Vermillion Border: Concave arc (Type 4).
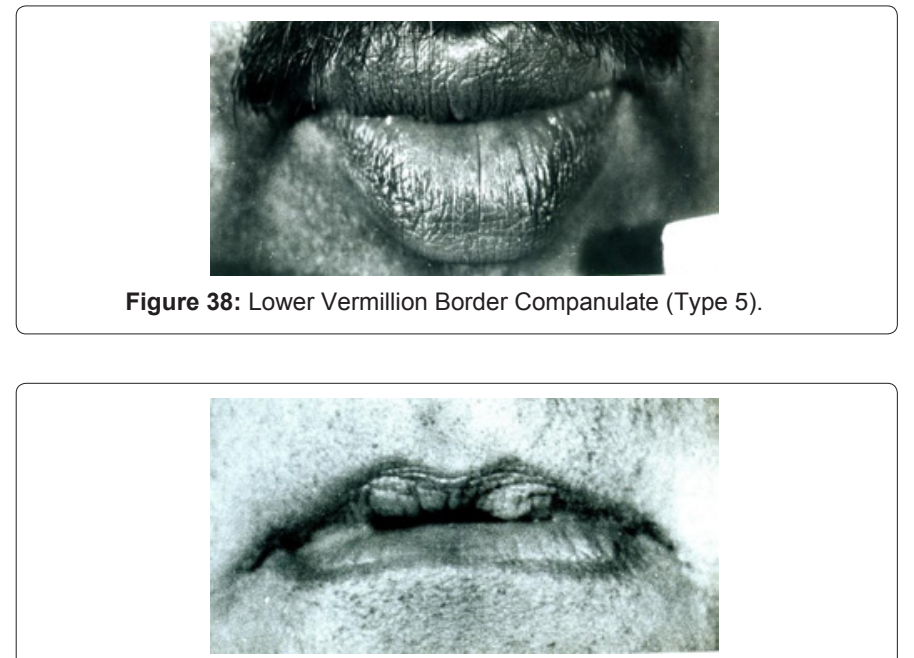

Figure 39: Lower Vermillion Border Flat (Type 6).

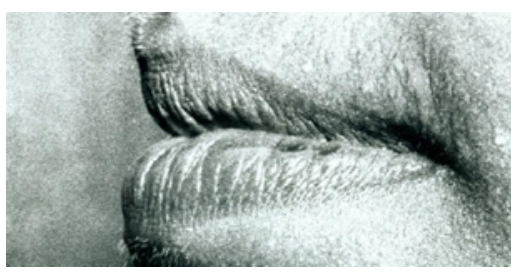

Figure 40: Protrusion: Even (Type 1)

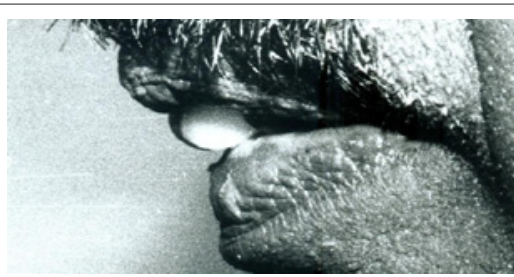

Figure 41: Protrusion: Protruding upper (Type 2).

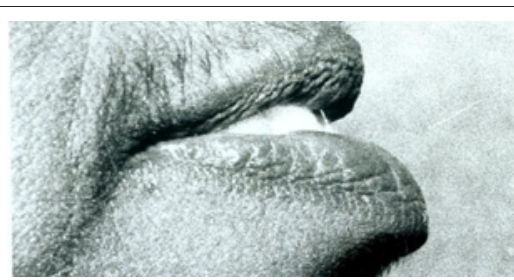

Figure 42: Protrusion Protruding lower (Type 3).

\section{Conclusion}

The total number of variations in lip formation according to the macrostructure defined above, depends upon the individual number of types in each of the five labial parameters viz (i) four types of sizes of lips; (ii) ten types of shapes of the oral fissure; (iii) twelve types of shapes of the upper vermillion border; (iv) six types of shapes of the lower vermillion border and (v) three types according to the protrusion of the lips.

Depending upon the different types identified by this researcher in 
each of the above five parameters, the number of possible configurations works out to be $4 \times 10 \times 12 \times 6 \times 3$. For personal appearance identification, if witnesses are able to describe correctly the five parameters, searching for suspects could be narrowed down to as small as one in 8640 .

We have also found in our study that no sex distinction could be attributed based on the size or shape of the lips. As a corollary, we found that it is impossible to attribute sex based on the pattern of grooves and wrinkles. It was found that no region-wise or district-wise distinctions or prominences exist either in macrostructure patterns or microstructure types.

\section{References}

1. Brandt PW, Pappas GD (1960) AN ELECTRON MICROSCOPIC STUDY OF PINOCYTOSIS IN AMEBA : I. The Surface Attachment Phase. J Biophys Biochem Cytol 8: 675-687.

2. Kumari TR, Chandra Sekharan P (1993) $13^{\text {th }}$ International Congress of Anthropological and Ethnological Sciences.

3. Kumari TR, Chandra Sekharan P (2003) Lips for personal appearance identification and personal identification, Proceedings of the $8^{\text {th }}$ Congress of Indo-Pacific Association of Law, Medicine and Forensic Science, Manila, Philippines.
4. Mattoo RK (1993) Read my lips, The Indian Express, Bangalore Edition.

5. The Hindu (1993) Identifying suspects by their lips, Madras Edition.

6. Kasprazk J (1990) Possibilities of Cheiloscopy. Forensic Sci Int 46: 145-151

7. Tsuchihashi $Y$ (1974) Studies on personal identification by means of lip prints Forensic Sci 3: 233-248.

8. Snyder L, Mulbar H, Wilson CM, Muehlberger CW (1944) Homicide investigation: practical information for coroners, police officers, and other investigators. Thomas Springfield, Illinois.

9. Santos M (2010) Queiloscopy -A supplementary stomatological means of identification. International Journal of Computer Applications 9: 10.

10. Suzuki K, Tsuchihashi $Y$ (1970) New attempt of personal identification by means of lip print. J Indian Dent Assoc 42: 8-9.

11. Saad WM, Kamel AH, Hassan FZ, EL-Otiefy MA (2005) Genetic Studies on the Inheritance of Lip Prints in-Cleft Lip and Palate. Egypt, J Plast Reconstr Surg 29: 9-12.

12. Tsuchihashi $Y$ (1974) Studies on personal identification by means of lip prints. Forensic Sci 3:233-248.

13. Castelló A, Alvarez-Seguí M, Verdú $F$ (2005) Luminous lip-prints as crimina evidence. Forensic Sci Int 155: 185-157.

14. Chandra Sekharan $P$ (1985) Identification of skull from its suture pattern. Forensic Sci Int 27: 205-214. 